\title{
The Assessment of Left Ventricle Function and Subclinical Atherosclerosis in Patients with Acute Myeloid Leukemia
}

\author{
ANDA MILITARU ${ }^{1}$, ADINA AVRAM $^{1}$, ANCA MARIA CIMPEAN $^{2}$, MIRCEA IURCIUC $^{3}$, \\ PETRU MATUSZ $^{4}$, DANIEL LIGHEZAN ${ }^{1}$ and MARIUS MILITARU ${ }^{5}$

\begin{abstract}
${ }^{1}$ Medical Semiology I, Department of Internal Medicine I, ${ }^{2}$ Department of Microscopic Morphology/Histology,
${ }^{4}$ Department of Anatomy and Embryology, and ${ }^{5}$ Neurology, Department of Neuroscience Municipal Emergency Hospital, Victor Babes University of Medicine and Pharmacy, Timisoara, Romania
\end{abstract} \\ Angiogenesis Research Center, ${ }^{3}$ Department of Cardiology, Preventive Medicine and Cardiovascular Rehabilitation,
}

\begin{abstract}
Aim To assess the onset of early left ventricular $(L V)$ systolic and diastolic function impairment and the subclinical atherosclerosis following chemotherapy in patients diagnosed with acute myeloid leukemia (AML). Materials and Methods: Thirty patients diagnosed with AML with no cardiac history, having $L V$ ejection fraction $(L V E F)>50 \%$, were evaluated at baseline and 6 months after starting four cycles of chemotherapy. We measured LV function, global longitudinal strain and subclinical atherosclerosis markers: intima-media thickness (IMT), arterial stiffness aortic pulse wave velocity (PWVAo) and ankle-brachial index (ABI). Results: LVEF had decreased at 6 months after treatment initialization $(p<0.001)$, the same changes being observed for $L V$ fraction shortening $(p<0.001)$, mitral annular plane systolic excursion and $S$ ' wave $(p<0.001$ and $p<0.05)$. Bilateral IMT and PWVAo significantly increased, 12 out of 30 patients (40\%) had LVEF $\leq 50 \%$ after 6 months of chemotherapy, five of them receiving daunorubicin at more than $500 \mathrm{mg} / \mathrm{m}^{2} /$ injection. Conclusion: LV function is impaired after 6 months of chemotherapy, with early changes of subclinical atherosclerosis becoming evident.
\end{abstract}

Cardiac toxicity represents one of the main side-effects of chemotherapy. One of the most alarming cardiotoxic effects is represented by left ventricular (LV) dysfunction, therefore

This article is freely accessible online.

Correspondence to: Anca Maria Cimpean, MD, Ph.D., Professor of Histology, Department of Microscopic Morphology/Histology, Angiogenesis Research Center, Piata Eftimie Murgu 2, 300041 Timisoara, Romania. Tel: +40 720060955, e-mail: ancacimpean1972@ yahoo.com

Key Words: Chemotherapy, cardiotoxicity, myocardial dysfunction, arterial stiffness, subclinical atherosclerosis, LV function. it would be useful to be able to detect cardiac dysfunction even in subclinical stages, before heart failure becomes clinically manifested. Anthracyclines and anthracenedione (mitoxantrone) are the most frequently used chemotherapeutic agents, with notable antitumor effects but they are also associated with high incidence of cardiotoxicity. Cardiotoxicity is associated with cumulative doses of the drug (1). Higher doses of chemotherapy are associated with higher probability of developing myocardial dysfunction and heart failure. Some patients often require high doses or several cycles of chemotherapy, leading to an increased cardiovascular risk $(1,2)$. Assessment of cardiac history and cardiovascular risk factors in patients undergoing chemotherapy is very useful in order to prevent or delay progression towards heart failure. Some side-effects of chemotherapy can occur immediately after therapy initialization, others can develop many years after treatment discontinuation (2). Patients undergoing radiation can develop not only cardiac dysfunction, but also vascular wall impairment, mainly at the level of the carotid arteries, particularly when radiation involves the thorax and cervical region (2). For these reasons, echocardiography and arterial stiffness measurement should be performed at baseline in all patients indicated for chemotherapy and repeated during the treatment and for a few years after the last dose $(1,3)$. The aim of the present retrospective study was to assess the onset of early signs of impairment of systolic and diastolic LV function and the subclinical atherosclerosis process following chemotherapy in patients diagnosed with acute myeloid leukemia (AML), since early therapeutic intervention is the only effective strategy in preventing or delaying the development of heart failure.

The purpose of our study was to identify the premature onset of myocardial dysfunction using echocardiographic parameters of LV function and detect the earliest signs of subclinical atherosclerosis using arterial parameters intima- 
media thickness (IMT), pulse wave velocity (PWV) and ankle brachial index (ABI) following 6 months of ' $3+7$ ', chemotherapy protocol.

\section{Materials and Methods}

Thirty patients diagnosed with AML, admitted to the hematology clinic, were enrolled. Inclusion criteria were: Patients diagnosed with AML with no previous history of cardiovascular diseases, left ventricular ejection fraction (LVEF) $>50 \%$, with no use of cardioprotective drugs (aspirin, angiotensin-converting enzyme inhibitor, beta blockers, angiotensin receptor blockers, nitrates) and without previous chemotherapy or radiotherapy. Patients received treatment according to current recommendations of the European Haematology Association (4). All standards in regard to ethics and good clinical practice were followed throughout our study. For all patients, clinical and echocardiographic evaluation were performed at baseline, before starting chemotherapy and after 6 months of therapy. Patients received chemotherapy treatment according to the ' $3+7$ ' protocol with daunorubicin ( 3 days) and cytarabine ( 7 days), repeated four times during the study duration. Clinical assessment consisted of: Medical history, and clinical examination, with the following parameters being registered: body mass index (BMI), heart rate (HR), systolic blood pressure (SBP), and diastolic blood pressure. Paraclinical assessment consisted of: Blood tests summarized in Table I, resting electrocardiogram (ECG), transthoracic echocardiography, and arterial parameters of subclinical atherosclerosis (IMT, PWV, ABI).

Transthoracic echocardiography. This was performed using a General Electric Vivid E9 ultrasound system with a $5 \mathrm{MHz}$ transducer (GE Healthcare, Silverwater, Australia), using a two-dimensional (2D) method, transverse sections, in M-mode Doppler pulsed at lateral mitral annulus. Images from transverse section were obtained in the following views: parasternal long axis, short axis of LV and apex, apical long axis of the 4, 3 and/or 2 chambers. LV performance was assessed by measuring LVEF using planimetry; fractional shortening (FS) was analyzed in 2D M-mode. LV diastolic function was assessed by measuring transmitral flow, Doppler inflow signal in parallel alignment with mitral blood flow, in order to quantify the maximum velocity of the early-filling wave (E-wave), atrial late-filling wave (A-wave) and ratio of early-to late-filling waves (E/A ratio). Quantitative assessment of systolic and diastolic function was evaluated using tissue doppler imaging (TDI), measuring the following parameters: Peak systolic velocity of the wall at the level of lateral mitral annulus (S'), peak early diastolic velocity of the wall at the mitral annulus (E'), peak late diastolic velocity of the wall at the mitral annulus (A'), ratio of early to late diastolic velocity of the wall (E'/A' ratio) and transmitral to early diastolic velocity ratio (E/E' ratio). Using TDI, we assessed global longitudinal deformation of the segments of LV during contraction. Longitudinal deformation of myocardial fibers was quantified using $2 \mathrm{D}$ echocardiography in apical 2-chamber, 3-chamber, and 4-chamber views. All echocardiographic examinations were performed by the same examiner.

Parameters of subclinical atherosclerosis. IMT was evaluated using a General Electric Vivid E9 ultrasound system with a linear array transducer of $9 \mathrm{MHz}$ (GE Healthcare). Measurements were obtained in the distal common carotid artery (CCA), $1 \mathrm{~cm}$ from the flow divider, at the far-wall level; 10 measurements were performed for each patient and mean values were registered. PWV assessment was performed using an accredited medical device Medexpert $\operatorname{arteriograph}^{\mathrm{TM}}$ TL2 (TensioMed, Budapest, Hungary). This oscillometric technique is based on the registration of pulse wave signals at the level of the brachial artery, blocked for a few seconds using a blood pressure cuff. PWV is a parameter used to quantify arterial stiffness - increased values represent a strong predictor of future major cardiovascular events. PWV is calculated from the distance traveled by pulse pressure waves in the aorta (measured through the distance from the suprasternal notch to the pubic bone) and measured transit time. These data are sent wirelessly to a computer, which allows data processing. Using these measurements, vascular age can be calculated, which can be different from biological age, mainly if vascular damaged has occurred. The patient is placed in supine position, relaxed, in a silent room; the only slight discomfort is related to blood pressure measurement, lasting approximately 3-5 minutes. For ABI, a BP monitor and a Doppler device, for detecting venous flow SonoTraX Vascular Doppler, (Edan Instruments, Langen, Germany) were used. With the BP monitor, SBP at both arms was measured; using the Doppler device, the first beat at the brachial artery level was detected. The highest value was registered for ABI calculation. Then we detected systolic BP was detected as the first Doppler signal at the posterior tibial artery and pedis artery, at the level of the lower limbs. ABI was calculated for each inferior limb, as the ratio of lower limb BP and upper limb BP, taking into consideration the highest $\mathrm{BP}$ value among those measured at the posterior tibial artery and brachial artery, respectively.

Statistical analysis. Statistical analysis was performed using SPSS program version 20.0 (IBM Corp, Armonk, NY, USA). Results are expressed as mean values \pm standard deviation. Comparison of LV function, vascular parameters, blood tests, heart rate and blood pressure after 6 months was made using paired $t$-test. Values of $p<0.05$ were considered statistically significant.

\section{Results}

Patients enrolled in our study had mean age of $47.67 \pm 13.34$ years. Among them, 12 were females with mean age of $50.42 \pm 12.78$ years and 18 men with mean age of $45.83 \pm 13.75$ years. Ten patients were younger or already had 45 years old (33.3\%) and 20 were older than 45 years old $(66.7 \%)$. Four cycles of chemotherapy were administered according to the ' $3+7$ ' protocol (3 days of daunorubicin at doses of $505.30 \pm 61.82 \mathrm{mg} / \mathrm{m}^{2} /$ day $\left(\right.$ range $=405-643 \mathrm{mg} / \mathrm{m}^{2} /$ day) and 7 days of cytarabine at doses of $2,613.26 \pm 321.97 \mathrm{mg} / \mathrm{m}^{2} /$ day (range $=2,100-3,332 \mathrm{mg} / \mathrm{m}^{2}$ ) until the second cardiologicaI evaluation. Eleven patients from the study group $(36.7 \%)$ received daunorubicin doses greater than $500 \mathrm{mg} / \mathrm{m}^{2} /$ day.

In our study, we noted a statistically significant increase of D-dimers from baseline to 6 months after treatment initialization $(p<0.05)$. The erythrocyte sedimentation rate also increased significantly from the baseline value after 6 months from chemotherapy initialization. The modifications noted in the other biological parameters did not reach statistical significance at 6 months from treatment initialization compared to baseline (Table I). 
Table I. Biological parameters at baseline and 6 months after chemotherapy. Data are the mean standard deviation (SD).

\begin{tabular}{lccc}
\hline Parameter & Baseline & After 6 months & $p$-Value \\
\hline D-Dimer (ng/ml) & $285.96 \pm 73.78$ & $368.63 \pm 180.24$ & 0.010 \\
Hb $(\mathrm{g} / \mathrm{dl})$ & $11.53 \pm 2.62$ & $10.87 \pm 2.38$ & 0.073 \\
Hct $(\%)$ & $34.46 \pm 8.41$ & $33.31 \pm 7.19$ & 0.315 \\
ESR $(\mathrm{mm} / \mathrm{h})$ & $28.06 \pm 19.02$ & $30.80 \pm 19.70$ & 0.011 \\
Glucose $(\mathrm{mg} / \mathrm{dl})$ & $101.93 \pm 19.05$ & $114.03 \pm 43.95$ & 0.102 \\
Creatinine $(\mathrm{mg} / \mathrm{dl})$ & $0.94 \pm 0.44$ & $1.08 \pm 0.62$ & 0.229 \\
Urea $(\mathrm{mg} / \mathrm{dl})$ & $36.20 \pm 24.77$ & $44.56 \pm 30.10$ & 0.124 \\
ALT $(\mathrm{U} / \mathrm{l})$ & $43.90 \pm 65.40$ & $41.83 \pm 29.06$ & 0.843 \\
AST $(\mathrm{U} / \mathrm{l})$ & $42.36 \pm 48.92$ & $37.40 \pm 27.79$ & 0.489 \\
Total bilirubin (mg/dl) & $0.78 \pm 0.50$ & $0.80 \pm 0.56$ & 0.826 \\
Na (mmol/l) & $140.36 \pm 2.88$ & $140.63 \pm 3.64$ & 0.755 \\
K (mmo/l) & $4.15 \pm 0.61$ & $4.17 \pm 0.85$ & 0.902 \\
Fibrinogen (mg/dl & $418.26 \pm 105.96$ & $453.20 \pm 139.82$ & 0.150 \\
Uric acid (mg/dl) & $5.88 \pm 2.62$ & $6.34 \pm 2.86$ & 0.267 \\
\hline
\end{tabular}

Hb: Hemoglobin; Hct: hematocrit, ESR: erythrocyte sedimentation rate, AST: aspartate aminotransferase, ALT: alanine aminotransferase, Na: sodium, K: potassium.

Table II. Echocardiographic parameters of left ventricle systolic and diastolic dysfunction before chemotherapy (at baseline) and at 6 months after chemotherapy initialization.

\begin{tabular}{lclc}
\hline Parameter & Baseline & After 6 months & $p$-Value \\
\hline IVS $(\mathrm{mm})$ & $9.7 \pm 1.4$ & $10.4 \pm 1.3$ & 0.001 \\
LVPW $(\mathrm{mm})$ & $9.7 \pm 1.2$ & $10.6 \pm 1.3$ & $<0.001$ \\
LVEDD $(\mathrm{mm})$ & $47.7 \pm 6.00$ & $50.30 \pm 6.01$ & $<0.001$ \\
LVESD $(\mathrm{mm})$ & $25.50 \pm 6.40$ & $28.30 \pm 7.4$ & $<0.001$ \\
LVEF $(\%)$ & $60.57 \pm 8.75$ & $52.93 \pm 8.70$ & $<0.001$ \\
LVESV $(\mathrm{ml})$ & $30.03 \pm 13.64$ & $37.16 \pm 14.83$ & $<0.001$ \\
LVEDV $(\mathrm{ml})$ & $76.23 \pm 24.47$ & $79.06 \pm 24.26$ & 0.268 \\
LVFS $(\%)$ & $43.10 \pm 12.49$ & $36.80 \pm 12.22$ & 0.001 \\
MAPSE $(\mathrm{mm})$ & $15.33 \pm 3.55$ & $13.06 \pm 3.60$ & $<0.001$ \\
\hline
\end{tabular}

IVS: Interventricular septum, LV: left ventricular; PW: posterior wall, FS: fractional shortening, EDD: end diastolic diameter, ESD: end systolic diameter, EF: left ventricular ejection fraction, EDV: end diastolic volume, ESV: end systolic volume, MAPSE: mitral annular plane systolic excursion.

Using 2D echocardiography at baseline and after 6 months from chemotherapy initialization (Figure 1), we observed statistically significant decrease of the following parameters in our study group: LVEF ( $p<0.001)$, LVFS $(p<0.001)$, and mitral annular plane systolic excursion (MAPSE) $(p<0.001)$. Significant changes were observed for other echocardiographic parameters: LV end-diastolic diameter, LV end-systolic diameter, and LV end-diastolic volume increased statistically significantly after 6 months compared to baseline $(p<0.001)$. A slight increase of interventricular septum $(p=0.001)$ and LV
Table III. Assessment of diastolic transmitral flow and left ventricle systolic function using pulsed wave tissue doppler imaging at the lateral mitral annulus, at baseline and at 6 months after chemotherapy initialization.

\begin{tabular}{lrrr}
\hline Parameter & Baseline & After 6 months & $p$-Value \\
\hline E $(\mathrm{m} / \mathrm{s})$ & $0.85 \pm 0.24$ & $0.84 \pm 0.23$ & 0.712 \\
$\mathrm{~A}(\mathrm{~m} / \mathrm{s})$ & $0.79 \pm 0.26$ & $0.82 \pm 0.24$ & 0.251 \\
E/A & $1.13 \pm 0.40$ & $1.08 \pm 0.37$ & 0.220 \\
$\mathrm{~S}^{\prime}(\mathrm{cm} / \mathrm{s})$ & $13.10 \pm 2.95$ & $11.80 \pm 3.14$ & 0.014 \\
$\mathrm{~A}^{\prime}(\mathrm{cm} / \mathrm{s})$ & $10.57 \pm 3.59$ & $9.37 \pm 2.93$ & 0.004 \\
$\mathrm{E}^{\prime}(\mathrm{cm} / \mathrm{s})$ & $11.90 \pm 4.30$ & $13.17 \pm 4.73$ & 0.019 \\
$\mathrm{E}^{\prime} / \mathrm{A}^{\prime}$ & $1.30 \pm 0.84$ & $1.58 \pm 0.97$ & 0.045 \\
$\mathrm{E}^{\prime}$ & $7.78 \pm 4.30$ & $6.95 \pm 3.38$ & 0.163 \\
\hline
\end{tabular}

E: Peak early diastolic trans mitral flow velocity, A: peak late diastolic trans mitral flow velocity, S': peak mitral annulus systolic velocity, E': peak early mitral annular diastolic velocity, A': peak late mitral annular diastolic velocity, E/A: ratio of maximum velocity of diastolic filling and maximum velocity of late diastolic filling through atrial contraction, E'/A': ratio of early mitral annular diastolic velocity and late mitral annular diastolic velocity, E/E': ratio of maximum velocity of diastolic filling and diastolic relaxation.

posterior wall $(p<0.001)$ in our study population was also noted at the end of the study (Table II).

In our study, 12 patients $(40 \%)$ had LVEF $\leq 50 \%$ after 6 months from chemotherapy initialization, LVEF decreased statistically significantIy from baseline to 6-month assessment (by $\sim 17 \%$; $p<0.001$ ) (Figure 2). LVSF also decreased statistically significant $(p<0.05)$. Among the 12 patients with LVEF $\leq 50 \%$ after 6 months, five had received daunorubicin in doses exceeding $500 \mathrm{mg} / \mathrm{m}^{2} /$ day.

We noted that changes of LV diastolic dysfunction parameters analyzed through transmitral flow - E-wave, Awave, E/A ratio, did not statistically significantly alter 6 months from treatment initialization compared to baseline. Assessment of pulsed wave TDI at the lateral mitral annulus parameters revealed that $S^{\prime}$ declined significantly $(p<0.05)$. E', A' and E'/A' ratio registered statistically significant variations in our study population after 6 months, increasing, decreasing and increasing $(p<0.05, p<0.05$ and $p<0.045)$, respectively (Table III). A statistically significant reduction of body weight in the whole study group after 6 months was noted, leading to a decrease of BMI $(p<0.05)$.

Regarding hemodynamic parameters, statistically significant changes were observed only in regard to HR, which increased from baseline $(p<0.05)$ after 6 months, whereas BP variations did not reach statistical significance. IMT registered a significant increase at the end of the study compared to baseline $(p<0.001)$, at the bilateral level. ABI decreased at both sides after 6 months, without reaching statistical significance. PWVAo increased statistically significantly 
Table IV. Assessment of hemodynamic and vascular parameters and body mass index (BMI) at baseline and 6 months after chemotherapy initialization.

\begin{tabular}{lccc}
\hline Parameter & Baseline & After 6 months & $p$-Value \\
\hline BMI $\left(\mathrm{kg} / \mathrm{m}^{2}\right)$ & $25.77 \pm 4.04$ & $25.38 \pm 4.01$ & 0.017 \\
HR $(\mathrm{bpm})$ & $79.76 \pm 10.22$ & $84.46 \pm 13.25$ & 0.035 \\
SBP $(\mathrm{mmHg})$ & $126.63 \pm 11.12$ & $128.63 \pm 14.61$ & 0.454 \\
DBP $(\mathrm{mmHg})$ & $77.23 \pm 10.83$ & $77.03 \pm 11.01$ & 0.941 \\
Left IMT $(\mathrm{mm})$ & $0.75 \pm 0.17$ & $0.82 \pm 0.16$ & $<0.001$ \\
Right IMT $(\mathrm{mm})$ & $0.74 \pm 0.17$ & $0.82 \pm 0.14$ & $<0.001$ \\
Left ABI & $1.11 \pm 0.11$ & $1.08 \pm 0.14$ & 0.265 \\
Right ABI & $1.13 \pm 0.23$ & $1.07 \pm 0.09$ & 0.142 \\
PVWAo (m/s) & $7.03 \pm 1.07$ & $7.97 \pm 1.12$ & $<0.001$ \\
Arterial age (years) & $37.37 \pm 11.16$ & $49.57 \pm 11.44$ & $<0.001$ \\
\hline
\end{tabular}

HR: Heart rate, SBP: systolic blood pressure, DBP: diastolic blood pressure, IMT: intima media thickness, ABI: ankle brachial index, PVWAo: pulse wave velocity in the aorta.

Table V. Speckle tracking assessment through global longitudinal strain (GLS) analyzed at baseline and 6 months after chemotherapy initialization.

\begin{tabular}{lccc}
\hline Parameter & Baseline & After 6 months & $p$-Value \\
\hline GLS-LAX (\%) & $-26.46 \pm 3.14$ & $-19.53 \pm 5.03$ & $<0.001$ \\
GLS-A4C (\%) & $-27.47 \pm 1.96$ & $-19.56 \pm 3.66$ & $<0.001$ \\
GLS-A2C (\%) & $-26.25 \pm 3.02$ & $-18.94 \pm 3.42$ & $<0.001$ \\
GLS-AVG (\%) & $-26.55 \pm 3.06$ & $-19.07 \pm 3.77$ & $<0.001$ \\
\hline
\end{tabular}

GLS-LAX: GLS of apical long axis, GLS-A4C: GLS of apical 4 chambers, GLS-A2C: GLS of apical 2 chambers, GLS-AVG: average GLS.

$(p<0.001)$, and a similar trend was noted in regard to arterial age $(p<0.001)$ (Table IV). In the 12 patients with $\mathrm{LVEF} \leq 50 \%$ at 6 months after chemotherapy initialization, vascular parameters registered similar trends to those of the whole study group: IMT $(p<0.05)$ and PWVAo $(p<0.001)$ increased.

Global longitudinal strain (GLS), quantified through Speckle tracking imaging, decreased statistically significantly after 6 months compared to baseline, from $-26.55 \pm-3.06 \%$ to $-19.07 \pm 3.77 \%(p<0.001)$ (Table V). GLS changes were noted all patients, without regard to sex: in male patients GLS was statistically significant reduced from $-26.09 \pm 3.56 \%$ at baseline to $-19.14 \pm 4.04 \%$ after 6 months from treatment initialization $(p<0.001)$, whereas in female patients, GLS decreased from $-27.25 \pm 2.04 \%$ at baseline to $-18.96 \pm 3.51$ $(p<0.001)$ at the end of our study. In the group of 12 patients with LVEF $\leq 50 \%$ after 6 months from therapy initialization, GLS significantly decreased compared to baseline from $-26.62 \pm 3.28 \%$ to $-18.13 \pm 4.54 \%(p<0.001)$ (Figure 3).

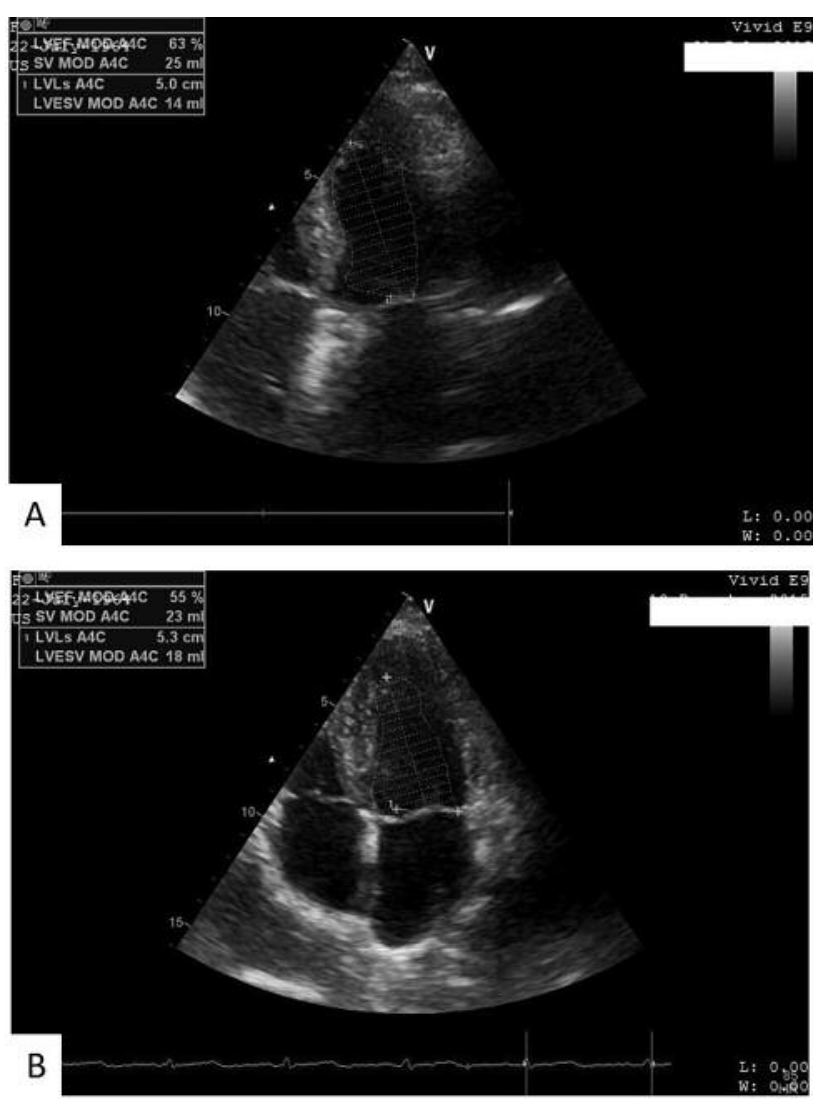

Figure 1. Assessment of left ventricular ejection fraction (LVEF) (\%) at baseline (A) and at 6 months after chemotherapy initialization (B) in a female patient with acute myeloid leukemia enrolled in our study. LVEF decreased by $8 \%$ at 6 months after chemotherapy treatment.

Out of the whole study group, 11 patients (36.7\%) received daunorubicin in doses above $500 \mathrm{mg} / \mathrm{m}^{2} /$ day. In these patients, a significant decrease was noted for both LVEF $(p<0.001)$ and MAPSE $(p<0.05)$. Speckle tracking imaging pointed out a significant reduction of GLS from $-26.85 \pm 2.17 \%$ at baseline to $-20.79 \pm 2.20 \%$ after 6 months $(p<0.001)$ (Figure 4). A significant deterioration was noted regarding subclinical atherosclerosis parameters after 6 months from therapy initialization compared to baseline: Right ABI decreased $(p<0.001)$, PWWAo increased $(p<0.001)$ and arterial age increased $(p<0.001)$.

\section{Discussion}

Acute leukemia represents a group of hematological malignancies with various immunological, molecular and clinical characteristics. Their treatment is based on cytostatic drugs which inhibit neoplastic cell growth and division. 

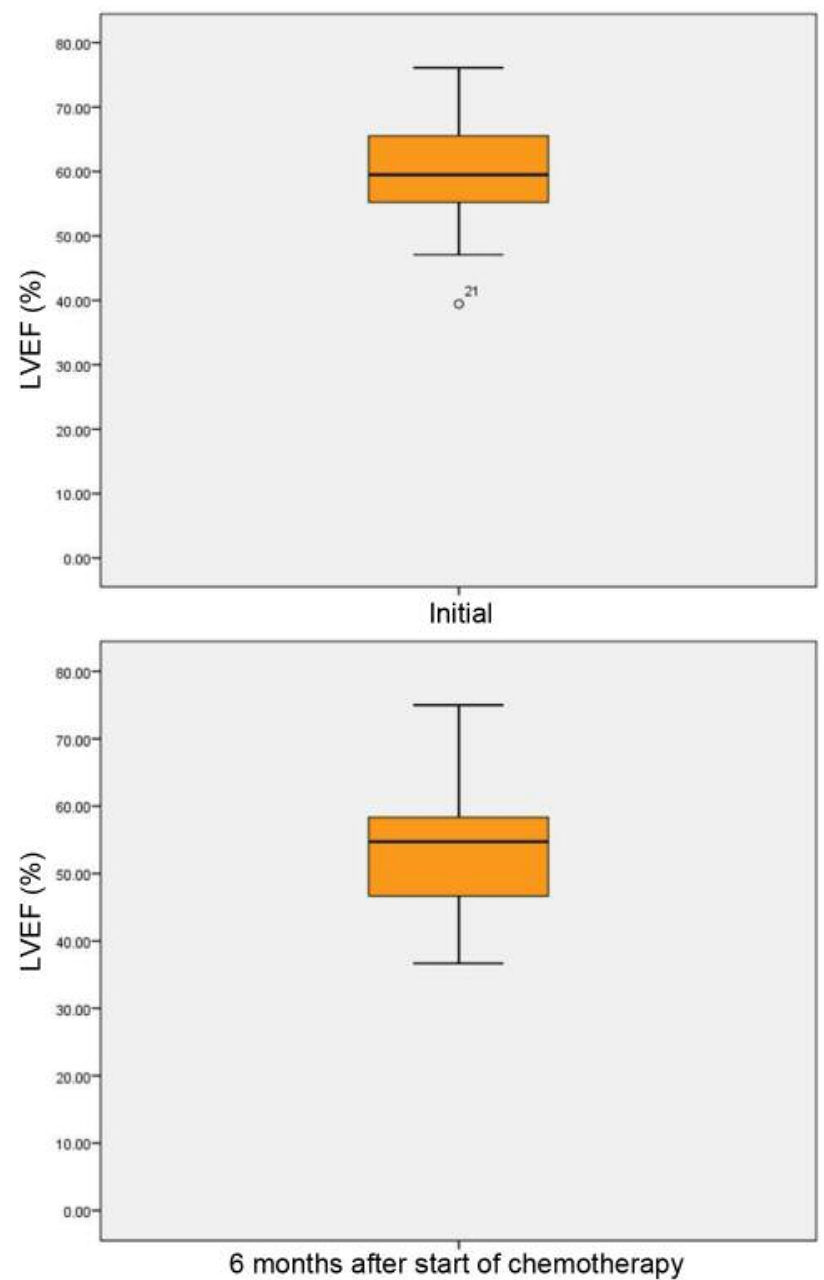

Figure 2. Left ventricular ejection fraction (LVEF) (\%) of patients with acute myeloid leukemia at baseline and 6 months after chemotherapy initialization. The line represents the median value, the box represents the interquartile range, and the bars represent the minimum and maximum values. Points outside the boxplot represent outlier values.

Effectiveness of cytostatic treatment also depends on patient age and comorbidities. Cardiotoxicity may represent the most serious complication/adverse effect of chemotherapeutic treatment $(3,5)$.

Our study group consisted of 30 patients diagnosed with AML. These patients received chemotherapy with daunorubicin as intravenous infusion over 20 minutes daily for 3 consecutive days and in combination with cytarabine in continuous intravenous infusion over 24 hours daily for 7 days, according to the ' $3+7$ ' protocol. Daunorubicin is an anthracycline and is a potent antileukemia drug. It can induce cardiotoxicity, a known side-
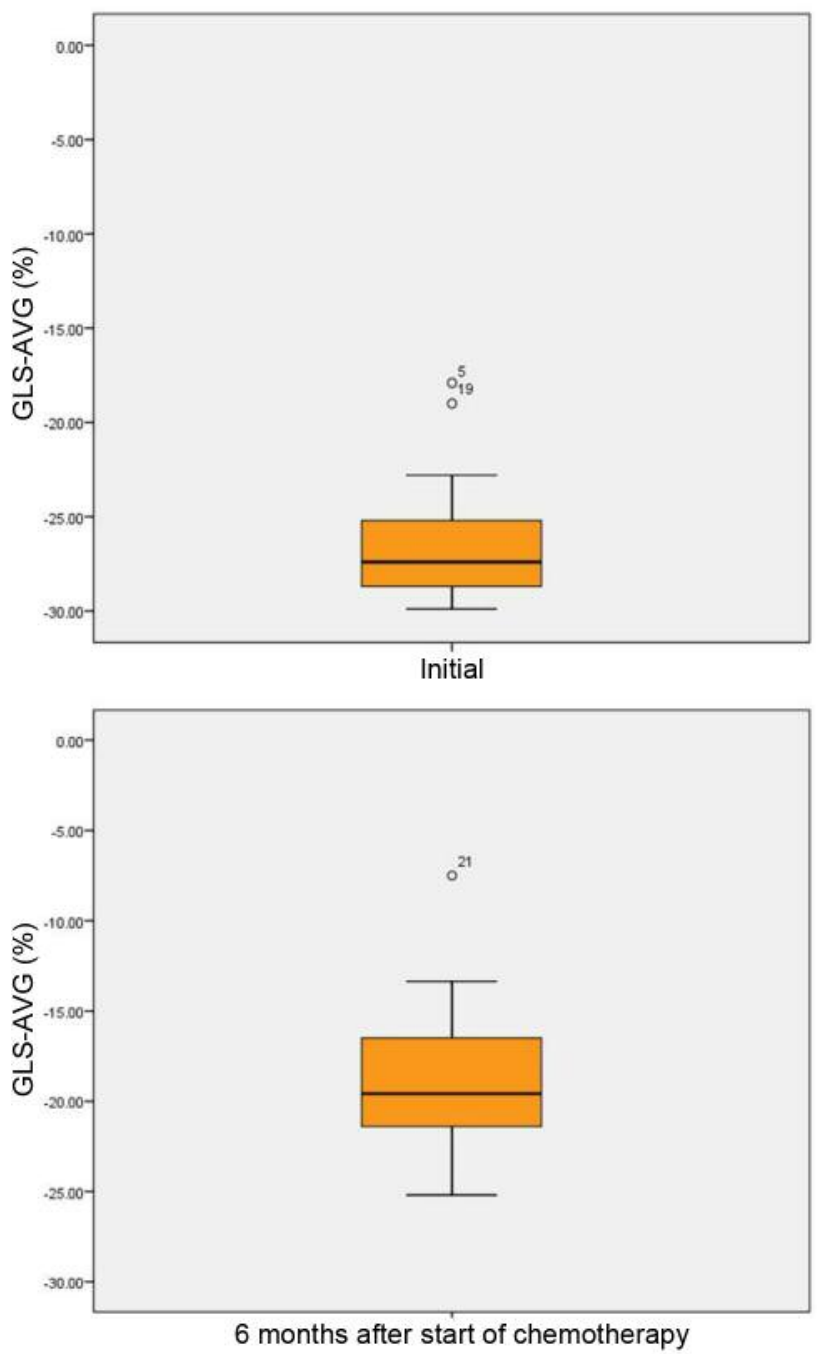

Figure 3. Speckle tracking: Average global longitudinal strain (GLS-AVG) (\%) of patients with acute myeloid leukemia at baseline and at 6 months after chemotherapy initialization. The line represents the median value, the box represents the interquartile range, and the bars represent the minimum and maximum values. Points outside the boxplot represent outlier values.

effect of anthracycline therapy. It has been established that a daunorubicin cumulative dose below $20 \mathrm{mg} / \mathrm{kg}$ is associated with a reduced cardiotoxicity risk, but higher doses imply an increased risk. Myocardial toxicity caused by daunorubicin can become clinical manifest during periods of treatment or later, months or years after administration. The highest cardiotoxicity risk is when daunorubicin doses are higher than $400-500 \mathrm{mg} / \mathrm{m}^{2}$ in adults (2). In clinical practice, high-doses of chemotherapeutic agent are often needed and even in association with other chemotherapeutic drugs or radiotherapy, leading to an increased risk of cardiotoxicity. Cytarabine, a bone-marrow 

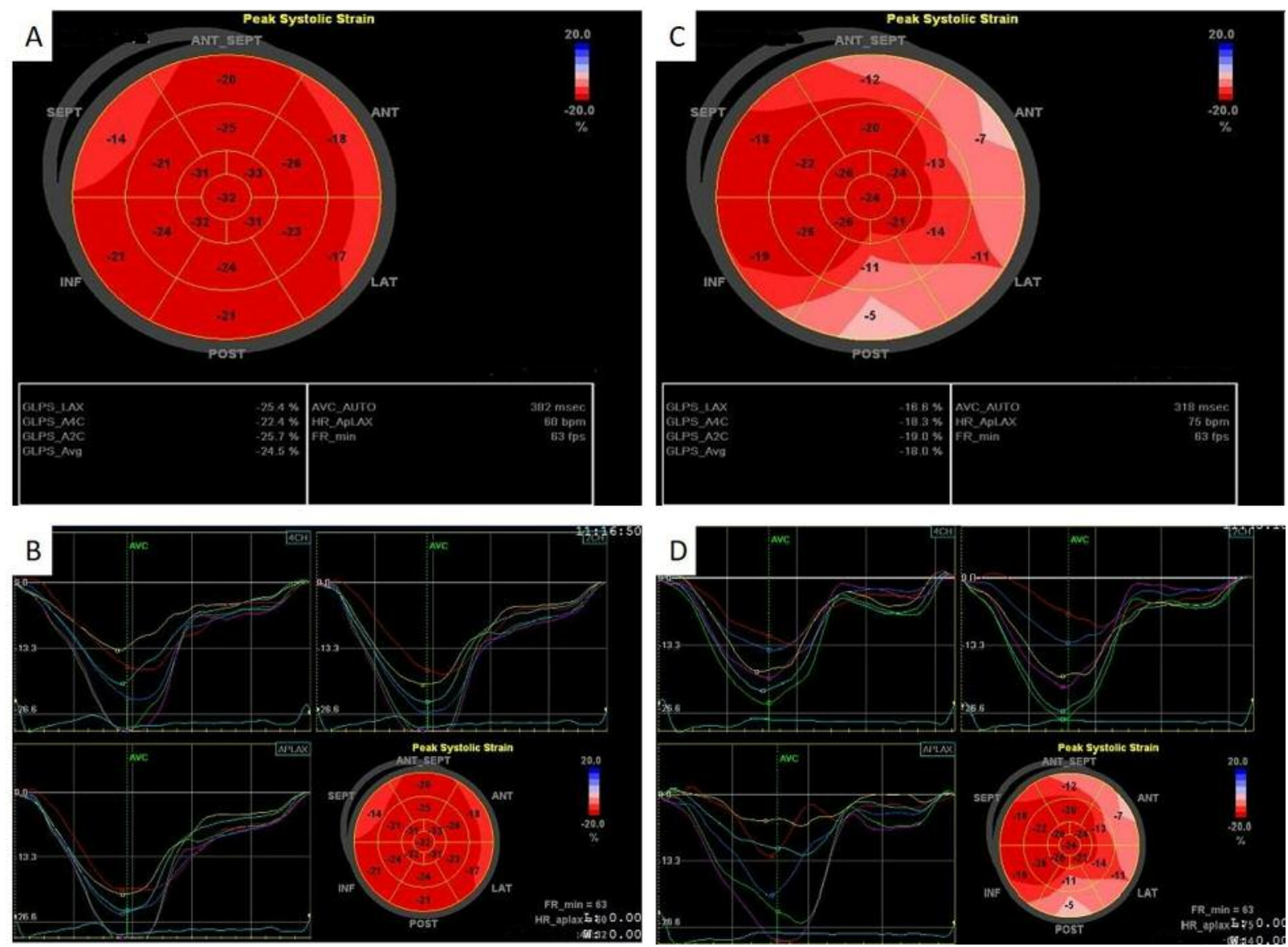

Figure 4. Speckle tracking: Global longitudinal strain (GLS) at baseline and at 6 months from chemotherapy initialization in a male patient with acute myeloid leukemia enrolled in our study. A, B: At the first determination, the values of the average GLS (GLS-AVG) (-24.5\%) were within the normal range. This shows that there were no changes in the structure and function of the left ventricle. C, D: After 6 months of chemotherapy, a decrease in the overall GLS-AVG (-18.0\%) was noted. This shows that there had been changes in myocardial structure from baseline. GLS-LAX: GLS of apical long axis, GLS-A4C: GLS of apical 4 chambers, GLS-A2C: GLS of apical 2 chambers.

suppressor, can affect cardiac function, mainly when it is associated with other cytostatics.

Many studies that assessed the cardiac toxicity of anthracycline have underlined the importance of LV function evaluation using echocardiography $(1,6)$. Echocardiography is an accessible, inexpensive, non-invasive investigatory technique, recommended to be performed before initialization of cytostatic treatment, periodically during treatment and long-term after chemotherapy $(1,2,7)$. In our study, echocardiography was performed at baseline, before chemotherapy initialization and at 6 months from initialization, after the fourth cycle of chemotherapy.

An echocardiographic parameter that can identify cardiac dysfunction early is the reduction of EF to below $50 \%$ with at least $10 \%$ reduction compared to baseline evaluation (1). Using 2D echocardiographic assessment, we noted that EF decreased statistically significantly after 6 months compared to baseline in our study. Among the 30 patients diagnosed with AML enrolled in the present study, 12 (40\%) had LVEF $\leq 50 \%$ after 6 months of chemotherapy. None of the 30 patients reported clinical symptoms of heart failure or severe arrythmia during the study period. Still, reduction of LVEF $\leq 50 \%$ indicates subclinical impairment of cardiac function. There are studies in the literature that showed that the reduction of LVEF represents a marker of cardiac toxicity even if it is not accompanied by clinical signs of heart failure $(2,8,9)$. In an important study which enrolled 2,625 patients treated with anthracycline, it was shown that only $9 \%$ of the patients reported clinical manifestation of cardiac toxicity immediately after chemotherapy. Most of these patients reported heart failure symptoms after at least 1 year following treatment administration (10). 
FS) is also an indicator of global systolic function, which provides information regarding radial and circumferential strain of the LV. In our study, FS decreased statistically significantIy 6 months from treatment initialization. MAPSE is an indicator of longitudinal and global systolic strain of LV. This echocardiographic parameter suffered modification compared to baseline.

There are studies showing the decrease of longitudinal strain is the strongest (negative) predictor in the development of cardiac toxicity following anthracycline in patients diagnosed with acute leukemia, lymphomas and other cancer types $(11,12)$ A GLS decrease of $11 \%$ compared to baseline suggests a deterioration of LV function, irrespective of the presence of clinical signs of heart failure $(2,12-14)$. In our study, we obtained results comparable with those existing in the literature. GLS decreased statistically significantly at 6 months from chemotherapy initialization compared to baseline in all patients enrolled in our study. This modification was noted not only in the 12 patients with LVEF $\leq 50 \%$ after 6 months from chemotherapy initialization, but also in those with LVEF $>50 \%$ after treatment.

Even though GLS represents a sensitive parameter in detecting cardiac toxicity, there are studies that underline that chemotherapy administration should not be interrupted when GLS decrease is not accompanied by other signs of heart failure (2). A meta-analysis on 159 patients receiving cytostatic treatment including anthracycline, demonstrated significant change of GLS in 52 patients (33\%), although only 14 patients experienced a reduction of LVEF by more than $10 \%$ compared to baseline. (15)

Comparable with GLS, S' wave measured through tissue Doppler can be an important predictor in early detection/identification of impairment of LV systolic function (2). Katarzyna et al. used tissue Doppler to asses 35 females with breast cancer who received anthracycline treatment and demonstrated that the only TDI parameter associated with impairment of LV systolic function was S' wave. This parameter decreased during chemotherapy (16). In our study, we obtained similar results for the patients enrolled. S' Wave decreased statically significantly after treatment compared to baseline. We also noted significant changes in other TDI parameters: E' increased, A-wave decreased, and E/E' ratio decreased, whereas E'/A' ratio increased 6 months from treatment initialization.

Some data presented in the literature showed that anthracyclines not only influence LV systolic and diastolic function but they also have an impact on parameters of arterial remodeling, leading to an increase of arterial stiffness, afterload and consequently to a reduction of coronary perfusion (17). In our study, the assessment of arterial stiffness performed at baseline and 6 months after treatment initialization provided data similar to those presented in the literature. We obtained a significant increase of PWVAo and IMT after the four cycles of chemotherapy, whereas ABI had decreased at the end of the study. Drafts et al. found a correlation between the reduction of LVEF and the increase of PWVAo at 6 months after chemotherapy in patients with leukemia, lymphomas or breast cancer (18). In a study that evaluated prognostic criteria and treatment for 85 patients with over $70 \%$ severe carotid stenosis, candidates for surgery or percutaneous carotid reperfusion, the risk of suffering a heart attack according to risk factors was statistically significant increased in patients with LVEF $\leq 50 \%$ compared to those with $\mathrm{LVEF}>50 \%$ (OR $=16.34,95 \%$ $\mathrm{CI}=4.58$ to $58.29, p=0.001)(19)$.

In another study, it was shown that for the assessment of subclinical atherosclerosis and onset of vascular aging, there should be a clear correlation between microscopic and clinical criteria (20). Carotid IMT and PVW are important indicators of subclinical atherosclerosis, risk assessment development of cardiovascular, cerebrovascular and target organ damage (21).

In a study comparing 82 children aged 1 to 14 years with central nervous system (CNS) tumors, with 110 patients of the same age group with leukemia, there was a statistically significant increase in IMT following chemotherapy in patients with CNS tumors (22). In another study evaluating the relationship between different types of cancer and cardiovascular atherosclerosis, an inverse correlation between tumors and atherosclerosis was reported. This correlation was not uniformly associated with antineoplastic treatment, suggesting that this inverse relationship may be partially attributable to intrinsic inflammation of a patient or to the modulating inflammatory properties of neoplasms (23).

Among biological parameters evaluated in our study, D-dimers and erythrocyte sedimentation rate were statistically significantly modified after 6 months compared to baseline among the 30 patients with AML enrolled. DDimers are considered markers of hypercoagulability status and of endogenous fibrinolysis. D-Dimers can be considered independent risk factors of cardiovascular mortality and can be useful in stratifying risk for heart failure (24).

\section{Conclusion}

In the present study, we demonstrated early deterioration of echocardiographic parameters of LV function in patients with AML following chemotherapy. GLS underlines modifications of LV function even at a subclinical stage in patients with AML. For this reason, we consider speckle tracking a useful tool in the cardiovascular assessment of patients with AML assigned to chemotherapy.

Parameters of subclinical atherosclerosis assessed in our study registered modifications in patients with AML following chemotherapy, therefore we consider the evaluation of arterial stiffness (PWV) and carotid ultrasound parameters (IMT) important in clinical practice. 
It is important following chemotherapy for patients with AML to perform periodic cardiovascular evaluations in order to be able to identify early vascular modifications and LV dysfunctions.

These data show that deterioration in myocardial fibers occurs starting with the first doses of chemotherapy; this deterioration is irreversible and can last months to years until the first signs of heart failure are reported by the patient. We believe cardiological assessment prior to starting chemotherapy should be compulsory for all patients diagnosed with AML; after chemotherapy, cardiological check-ups should be performed periodically, for a long period of time after finishing chemotherapy.

\section{Conflicts of Interest}

None declared.

\section{Acknowledgements}

The present work was developed with the support of Atherosclerosis and Atherothrombosis Group of Romanian Society of Cardiology and support of Victor Babes University of Medicine and Pharmacy Timisoara, Romania.

\section{References}

1 Cardinale D, Colombo A, Lamantia G, Colombo N, Civelli M, De Giacomi G, Rubino M, Veglia F, Fiorentini C and Cipolla $\mathrm{CM}$ : Anthracycline-induced cardiomyopathy: clinical relevance and response to pharmacologic therapy. J Am Coll Cardiol 55(3): 213-220, 2010.

2 Zamorano JL, Lancellotti P, Muñoz DR, Aboyans V, Asteggiano R, Galderisi M, Habib G, Lenihan DJ, Lip GY, Lyon AR, Fernandez TL, Mohty D, Piepoli MF, Tamargo J, Torbicki A and Suter TM: 2016 ESC Position Paper on cancer treatments and cardiovascular toxicity developed under the auspices of the ESC Committee for Practice Guidelines. Eur Heart J 37(36): 2768-2801, 2016.

3 Simon A, Chironi G and Levenson J: Comparative performance of subclinical atherosclerosis tests in predicting coronary heart disease in asymptomatic individuals. Eur Heart J 28(24): 29672971, 2007.

4 Döhner H, Estey EH, Amadori S, Appelbaum FR, Büchner T, Burnett AK, Dombret H, Fenaux P, Grimwade D, Larson RA, Lo-Coco F, Naoe T, Niederwieser D, Ossenkoppele GJ, Sanz MA, Sierra J, Tallman MS, Löwenberg B and Bloomfield CD: Diagnosis and management of acute myeloid leukemia in adults: recommendations from an international expert panel, on behalf of the European Leukemia Net. Blood 115(3): 453-474, 2010.

5 Jain D, Russell RR, Schwartz RG, Panjrath GS and Aronow W: Cardiac complications of cancer therapy: Pathophysiology, identification, prevention, treatment, and future directions. Curr Cardiol Rep 19(5): 36, 2017.

6 Jurcut R, Wildiers H, Ganame J, D'hooge J, Paridaens R and Voigt JU: Detection and monitoring of cardiotoxicity-What does modern cardiology offer. Supportive Care in Cancer 16(5): 437$445,2008$.
7 Stellitano A, Fedele R, Barilla S, Iaria A, Rao CM and Martino M: Chemotherapy and cardiotoxicity in hematologic malignancies. Curr Cancer Drug Targets 17(4): 311-324, 2017.

8 Plana JC, Galderisi M, Barac A, Ewer MS, Ky B, ScherrerCrosbie M, Ganame J, Sebag IA, Agler DA, Badano LP, Banchs J, Cardinale D, Carver J, Cerqueira M, DeCara JM, Edvardsen T, Flamm SD, Force T, Griffin BP, Jerusalem G, Liu JE, Magalhaes A, Marwick T, Sanchez LY, Sicari R, Villarraga HR and Lancellotti P: Expert consensus for multimodality imaging evaluation of adult patients during and after cancer therapy: a report from the American Society of Echocardiography and the European Association of Cardiovascular Imaging, Eur Heart J Cardiovasc Imaging 15: 1063-1093, 2014.

9 Lang RM, Badano LP, Mor-Avi V, Afilalo J, Armstrong A, Ernande L, Flachskampf FA, Foster E, Goldstein SA, Kuznetsova T, Lancellotti P, Muraru D, Picard MH, Rietzschel ER, Rudski L, Spencer KT, Tsang W and Voigt JU: Recommendations for cardiac chamber quantification by echocardiography in adults: an update from the American Society of Echocardiography and the European Association of Cardiovascular Imaging. J Am Soc Echocardiogr 28: 1-39.e14, 2015.

10 Cardinale D, Colombo A, Bacchiani G, Tedeschi I, Meroni CA, Veglia F, Civelli M, Lamantia G, Colombo N, Curigliano G, Fiorentini $\mathrm{C}$ and Cipolla CM: Early detection of anthracycline cardiotoxicity and improvement with heart failure therapy. Circulation 131: 1981-1988, 2015.

11 Thavendiranathan P, Poulin F, Lim KD, Plana JC, Woo A and Marwick TH: Use of myocardial strain imaging by echocardiography form the early detection of cardiotoxicity in patients during and after cancer chemotherapy: a systematic review. J Am Coll Cardiol 63(25 Pt A): 2751-2768, 2014.

12 Boyd A, Stoodley P, Richards D, Hui R, Harnett P, Vo K, Marwick T and Thomas L: Anthracyclines induce early changes in left ventricular systolic and diastolic function: A single-centre study. PLoS One 12(4): e0175544, 2017.

13 Sawaya H, Sebag IA, Plana JC, Januzzi JL, Ky B, Tan TC, Cohen V, Banchs J,Carver JR, Wiegers SE, Martin RP, Picard MH, Gerszten RE, Halpern EF, Passeri J, Kuter I and ScherrerCrosbie M: Assessment of echocardiography and biomarkers for the extended prediction of cardiotoxicity in patients treated with anthracyclines, taxanes, and trastuzumab. Circ Cardiovasc Imaging 5: 596-603, 2012.

14 Negishi K, Negishi T, Hare JL, Haluska BA, Plana JC and Marwick TH: Independent and incremental value of deformation indices for prediction of trastuzumab-induced cardiotoxicity. J Am Soc Echocardiogr 26: 493-498, 2013.

15 Negishi K, Negishi T, Haluska BA, Hare JL, Plana JC, and Marwick TH: Use of speckle strain to assess left ventricular responses to cardiotoxic chemotherapy and cardioprotection, Eur Heart J - Cardiovasc Imaging 15: 324-331, 2014.

16 Mizia-Stec K, Gościńska A, Mizia M, Haberka M, Chmiel A, Poborski W, Gąsior Z: Anthracycline chemotherapy impairs the structure and diastolic function of the left ventricle and induces negative arterial remodeling. Kardiologia Polska 7: 681-690, 2013.

17 Mihalcea DJ, Florescu M, Suran BM, Enescu OA, Mincu RI, Magda S, Patrascu N and Vinereanu D: Comparison of pulse wave velocity assessed by three different techniques: Arteriograph, Complior, and Echo-tracking. Heart Vessels 31(4): 568-577, 2016. 
18 Drafts BC, Twomley KM, D’Agostino R Jr., Lawrence J, Avis N, Ellis LR, Thohan V, Jordan J, Melin SA, Torti FM, Little WC, Hamilton CA and Hundley WG: Low to moderate dose anthracycline-based chemotherapy is associated with early noninvasive imaging evidence of subclinical cardiovascular disease. JAAC Cardiovasc Imaging 6(8): 877-885, 2013

19 Militaru M, Militaru A, Lighezan D, Petrescu L and Simu M: Prognostic criteria and treatment for patients candidates for surgery or percutaneous carotid reperfusion, Rom J Neurol 13(2): 60-68, 2014

20 Iurciuc S, Cimpean AM, Mitu F, Heredea R and Iurciuc M:Vascular aging and subclinical atherosclerosis: Why such a 'never ending' and challenging story in cardiology? Clin Interv Aging 12: 1339-1345, 2017.

21 Iurciuc S, Avram C, Turi V, Militaru A, Avram A, Cimpean AM and Iurciuc M: Physical training, hemodynamic parameters and arterial stiffness: friends or foes of the hypertensive patient? In Vivo 30(4): 521-528, 2016.
22 Dengel DR, Kelly AS, Zhang L, Hodges JS, Baker KS and Steinberger J: Signs of early sub-clinical atherosclerosis in childhood cancer survivors. Pediatr Blood Cancer 61(3): 532537, 2014.

$23 \mathrm{Li} \mathrm{M}$, Cima MJ and Milner DA Jr.: If it's not one thing, it's another: An inverse relationship of malignancy and atherosclerotic disease. PLoS One 10(5): e0126855, 2015.

24 Danesh J, Whincup P, Walker M, Lennon L, Thomson A, Appleby P, Rumley A, and Lowe GD: Fibrin D-dimer and coronary heart disease: Prospective study and meta-analysis. Circulation 103: 2323, 2001.

Received June 23, 2018

Revised July 29, 2018

Accepted August 7, 2018 